\title{
Anotaciones sobre Guastini
}

\author{
Michel Troper \\ Professeur émérite à l'Université de Paris Ouest Nanterre, \\ Membre de l'Institute Universitaire de France, Centre de Théorie \\ et Analyse du Droit - UMR CNRS 7074 \\ Traducción de Federico José Arena
}

Riccardo Guastini presenta en su nuevo artículo una reformulación extremadamente interesante y estimulante de la teoría escéptica de la interpretación. Guastini propone una versión moderada de esta teoría y descarta la versión radical a la que denomina, siguiendo a Hart, "teoría de la pesadilla".

Para quien, como yo, adhiere a la versión radical de la teoría escéptica, evidentemente no existen desacuerdos fundamentales respecto de las tesis de RG, sino solamente de matices. RG ha tenido ya la oportunidad de desarrollar sus ideas en otros escritos; sin embargo, en esta ocasión lleva a cabo una presentación novedosa, hábil y compleja, que hace que estos matices sean importantes, y que exige una discusión detallada.

Para proceder adecuadamente será necesario, antes de comenzar con la discusión, disponer de una definición de la tesis escéptica y de cada una de sus versiones, la moderada y la radical. En este sentido, he de señalar que la presentación que RG hace de la versión radical es una reconstrucción que, desde mi punto de vista, no se corresponde para nada con el modo de ver de los defensores de esa versión. En cuanto a la tesis moderada, ella en realidad no es expuesta de entrada, sino que, 
en cambio, aparece a lo largo de la argumentación. Ello requiere que el lector la reconstruya paso a paso.

La originalidad de la presentación se encuentra en el hecho de estar dividida en dos partes: una teoría y una metateoría.

Bajo el título "teoría", se describen y distinguen las diferentes operaciones que llamamos "interpretación" y las razones por las cuales se hacen estas distinciones; razones que tienen que ver con la indeterminación del derecho aplicable.

Bajo el título "metateoría" se examinan diferentes teorías de la interpretación, concebidas y reconstruidas como teorías semánticas. Estas teorías son objeto de una crítica detallada dirigida principalmente contra las teorías del significado que cada una de ellas presupone. Algunas de ellas presupondrían que el significado es cognoscible, otra, la teoría escéptica radical, que antes de la interpretación los textos jurídicos no poseen significado alguno.

Esta división entre teoría y metateoría, y sobretodo el orden de la presentación, poseen, creo, el siguiente inconveniente: para examinar los presupuestos de las teorías de la interpretación y criticarlos, sería necesario disponer de una definición de estas teorías que sea independiente de la propia teoría de la interpretación. Ahora bien, gran parte de las afirmaciones realizadas por RG en la segunda parte dependen directamente de las tesis enunciadas por RG en la primera. Por ejemplo, la idea según la cual la interpretación está ligada a, y resuelve las dudas producidas por, la indeterminación de los textos jurídicos, o la idea de que existe una diferencia entre interpretación y creación. De ello se sigue que el razonamiento es circular y deja la impresión de que las teorías criticadas en la segunda parte son solo culpables de contradecir las tesis enunciadas en la primera. En virtud de ello, esta segunda parte no se ubica en realidad en un nivel metateórico (excepto en cuanto se refiere a la teoría del significado), sino a un nivel simplemente teórico.

Dicho esto, podemos pasar a considerar los argumentos en detalle siguiendo el orden en el que son presentados. 


\section{Teoría}

En la parte "teoría", el autor comienza distinguiendo numerosos tipos de ambigüedad que afectan el término "interpretación". A continuación RG se refiere a aquello que hace que la interpretación sea necesaria, a saber, la indeterminación jurídica.

\subsection{Los tipos de ambigüedad}

a) La interpretación haría referencia algunas veces a la atribución de un significado a un texto jurídico y otras veces a la inclusión de un caso en una clase de casos regulados por una norma.

RG subraya que se trata de una distinción lógica, puesto que una cosa es determinar, en un primer momento, el significado de un texto y otra cosa es determinar si se aplica a un caso concreto.

El autor concede que, psicológicamente, quizás las cosas funcionen de manera diferente e incluso que quizás los jueces procedan inversamente, es decir, subsumiendo primero el caso bajo una categoría, y determinando luego el significado del texto. Pero ello consiste meramente en una descripción de los procesos psicológicos del juez. RG estima por lo tanto necesario distinguir entre la interpretación de los textos y la interpretación de los hechos.

Sin embargo, podemos objetar que las dos operaciones se confunden, y ello no solo, tal como admite el mismo RG, en el plano psicológico (el orden en el que procedemos es claramente irrelevante), sino mas bien en el plano lógico. Retomemos el ejemplo clásico de los vehículos sobre el que razona $R G$. ¿Una bicicleta es o no un vehículo? ¿Una filosofía newage es una religión? No es posible responder a estas preguntas examinando aquellos "hechos" que son bicicletas o que son la filosofía new-age. No podemos hacer otra cosa más que analizar las clases "vehículo" y "religiones" que preexisten a la clasificación. Todo depende efectivamente del significado de los términos "vehículo" o "religión" y sus significados son aquellos que son asociados a ciertos textos. Por otra parte, el juez podrá decir que la bicicleta es un vehículo en el sentido del texto que prohíbe a los vehículos entrar en un parque 
público, pero que ella no es un vehículo en el sentido del derecho tributario o en el sentido de la legislación sobre la responsabilidad por accidentes de tránsito. Respecto de la interpretación de la norma sobre el parque se trata de un vehículo, si llamamos "vehículo" a todo aquello que rueda y que permite trasportar personas. No es un vehículo si usamos ese término para referirnos a las cosas que pueden presentar un peligro dentro de un espacio destinado al paseo.

El derecho administrativo francés distingue, tal como lo hace RG, la interpretación de los textos y la clasificación de los hechos, a la denominada "calificación jurídica de los hechos", pero se trata simplemente de un intento para disimular el hecho que también en el segundo caso se interpretan textos. Ello puede verse usando el siguiente ejemplo: una ley de 1911 permitía a la administración rechazar un permiso para construir, si el inmueble proyectado era de una naturaleza tal que podía perjudicar una perspectiva monumental. Luego de que su pedido para construir en la plaza Beauvau de Paris fuera rechazado, el propietario del terreno atacó la decisión ante el Consejo de Estado. El Consejo de Estado la anuló sosteniendo que "la plaza Beauvau no podría ser considerada en su conjunto como formando parte de una perspectiva monumental" ". Eso es todo en lo que consiste la justificación de la sentencia y en ello reside precisamente el interés del juez de presentar de este modo las cosas. El juez finge examinar la plaza Beauvau y de descubrir su naturaleza simplemente observando hechos, todo lo cual le permite eludir la justificación de su decisión y de analizar el significado literal de los términos, o la intención del legislador, o las consecuencias económicas de la solución elegida. En realidad, lo que hace, es contribuir a dar un significado a la expresión "perspectiva monumental" contenida en el texto de la ley. Es por ello que los manuales prácticos destinados a los abogados incluyen a menudo una lista de casos, de manera tal que sea posible, a partir de esas listas, inferir el significado de los textos. La clase "perspectiva monumental" es definida por extensión y la expresión designa el conjunto de perspectivas que así han sido clasificadas en el pasado. 
Es cierto, tal como lo dice RG, que la calificación jurídica de los hechos no termina en enunciados de la forma "El texto T significa S", pero sería equivocado concluir sobre esta base que no se trata de una interpretación de un texto. Es necesario recordar que en el sistema francés se encuentra, desde 1790, oficialmente prohibido a los jueces interpretar la ley. Dado que no es posible aplicar la ley sin interpretarla, se ha debido presentar la interpretación como una "aplicación" y las decisiones judiciales han sido casadas por "errónea aplicación de la ley". Es decir, se trata de un vicio que en otros sistemas habríamos podido llamar "errónea interpretación de la ley". En efecto, si bien el dispositivo de la decisión no contiene un enunciado del tipo "El texto T significa S", la serie de fórmulas "La plaza Beauvau no es una perspectiva monumental", "La plaza X es una perspectiva monumental", "La plaza $\mathrm{Y}$ es una perspectiva monumental" permiten individuar mediante generalización los caracteres que conducirían a subsumir una plaza dentro de la clase de las perspectivas monumentales y, de este modo, construir la fórmula "La expresión 'perspectiva monumental' incluida en el texto designa una plaza que posee las características siguientes...".

Especialmente, las dos operaciones que distingue RG presentan dos características comunes esenciales, a saber, se trata de decisiones, y ellas no son consecuencia de la simple observación de los hechos.

Desde un punto de vista jurídico, se trata de decisiones. No poseen valor de verdad, pero, en tanto y en cuanto ellas emanan de una autoridad competente, se imponen jurídicamente. Del mismo modo en que es obligatorio aplicar la ley según el significado que el juez le ha dado al texto, es obligatorio tratar las bicicletas como vehículos si el juez ha decidido que son vehículos.

Por otro lado, la inclusión de un hecho en un clase depende de ciertas características, pero la lista de características que han de ser consideradas depende de una evaluación de la oportunidad de someter este hecho a un cierto régimen jurídico, tal como la elección de un método de interpretación de un texto. Asimismo, puede ser el caso que el "hecho" a incluir en una clase no sea un hecho material, sino un texto jurídico. Esto es lo que sucede cuando un tribunal constitucional examina la conformidad de una ley con la constitución. La conformidad depende 
naturalmente del significado asignado a la constitución y al mismo tiempo del significado asignado a la ley sujeta a control por parte del tribunal. Para poder "subsumir" es necesario determinar el significado de un texto y esta operación no es menos constructiva que la precedente.

b) la segunda ambigüedad proviene del hecho que, por "interpretación", se entiende a veces la identificación de los diferentes significados posibles de un texto, otras veces la elección por parte del juez de uno de esos significados $y$, otras veces, la atribución de un significado que no había sido identificado con anterioridad.

Antes de continuar, es necesario observar que el término ambigüedad es usado aquí en un sentido ligeramente distinto del párrafo precedente. Allí, se usaba para referirse a dos operaciones diferentes (atribución de significado e inclusión en una clase), ambas designadas por el lenguaje jurídico o por el lenguaje de la teoría del derecho con el mismo término ("interpretación"). Aquí, en cambio, se refiere unas veces a operaciones o funciones diferentes (conocer y decidir) y, otras veces, a la misma operación, pero concebida según teorías distintas. La ambigüedad en este caso no es un rasgo del término "interpretación", sino de la operación a la que el término se refiere, en cuanto esa operación es objeto de teorías rivales. En este sentido, el término "interpretación" no es más ambiguo que el término "ballena", que posee siempre la misma referencia aun cuando algunos creen que se trata de un pescado mientras otros prefieren incluir las ballenas dentro de la clase de los mamíferos. Es posible que algunos consideren que el juez, cuando lleva a cabo una "interpretación", elije entre varios significados posibles, mientras que otros consideren que el juez atribuye al texto un significado que el texto no poseía antes de ese momento, e incluso otros que (como RG) consideran que el juez hace a veces una cosa y a veces la otra.

Si se tratara de una tesis sobre el lenguaje, podríamos contentarnos con observar el lenguaje ordinario y verificar si posee esas ambigüedades, sin que sea necesario examinar si el lenguaje común se equivoca. Intentaríamos establecer, por ejemplo, si es verdad que en el lenguaje común se emplea el término "interpretación" para designar una operación que consideramos en realidad una creación. Probablemente la conclusión sería negativa. La mayor parte de quienes 
emplean el término piensan que la creación es algo diferente de la interpretación y se negarían a emplear el mismo término para referirse a ambas. Tal como el mismo Guastini afirma: "la interpretación creativa, tal como ha sido aquí definida, es un fenómeno algo raro" ${ }^{2}$. ¿Por qué afirmar entonces que las ambigüedades pertenecen al lenguaje común? En realidad, únicamente aquellos que adhieren a alguna versión de la teoría realista sostienen que la interpretación es, al menos en parte, una creación y aceptan emplear el término en este sentido.

De este modo, lo que RG sostiene aquí no es realmente una distinción de varios significados del término "interpretación" en el lenguaje jurídico ordinario, sino una distinción teórica que él considera útil hacer entre distintas actividades.

Es por lo tanto necesario analizar la pertinencia de esta distinción, a partir de las preguntas siguientes:

- ¿Es verdadero que la identificación de los significados posibles de un texto es un acto de conocimiento?

- ¿Existe alguna diferencia entre la interpretación decisoria y la interpretación creativa?

Acerca del primer punto es indiscutible que existe una actividad, llevada a cabo por los comentadores, los profesores de derecho, los abogados, y los jueces, que consiste en identificar los distintos significados posibles. Pero para que esta actividad pueda ser considerada como una actividad de conocimiento, sería necesario que estos significados posibles posean una determinada forma de existencia y que sea posible distinguir entre significados posibles y significados imposibles. Entiéndase bien, no es que esté prohibido denominar "significado posible" al significado que podemos determinar sobre la base de las reglas del lenguaje o los usos de una comunidad. Pero ipor qué un significado que pueda ser obtenido independientemente de las reglas del lenguaje, como por ejemplo mediante referencia a la función social de la norma, o a una doctrina jurídica, o a una ideología diferente de los usos extendidos en una comunidad, no sería un significado "posible"?

${ }^{2}$ Ref. a Guastini. 
¿No podríamos considerar como significado posible el que resulta de una interpretación conforme a la religión católica, al marxismo leninismo, o a la ideología nacional socialista? Si se respondiera afirmativamente, sería entonces necesario concluir que la lista de los significados posibles es infinita, porque no hay un significado tan alejado de las reglas del lenguaje y de los usos comunes, que no pueda ser asociado a una ideología cualquiera.

En cuanto a la diferencia entre la interpretación decisoria y la interpretación creativa, ella descansa sobre el presupuesto, bastante frágil como vimos, de que la lista de significados posibles no es infinita.

c) Bajo la rúbrica "tercera ambigüedad", RG extiende la distinción precedente entre interpretación y creación oponiendo la interpretación propiamente dicha (la atribución de un significado a un texto) y la construcción jurídica, es decir el conjunto de operaciones dirigidas a hacer aparecer normas no expresas. Por norma "no expresa" RG entiende una norma que no puede ser considerada como el significado de un texto dado y que ha sido obtenida, no a partir de procedimientos lógicos, sino sobre la base de razonamientos fundados en teorías arbitrarias.

Sin embargo, al igual que en el caso anterior, RG parece presuponer que la primer operación (la interpretación propiamente dicha) no es el producto de una construcción, como si el significado de los textos se encontrara directamente disponible al lector. Si se admite la distinción entre un enunciado y su significado, una distinción claramente esencial para la teoría guastiniana del derecho, entonces la distinción entre normas expresas y normas no expresas se desmorona. Una norma es expresa en tanto y en cuanto es considerada como el significado de un enunciado y solo puede serlo como resultado de un razonamiento interpretativo. Además, no existe ninguna diferencia entre la interpretación propiamente dicha y la construcción jurídica. Retomando su ejemplo del artículo 139 de la Constitución Italiana, los juristas que consideran que "la forma republicana del Estado" significa "democracia" seguramente están embarcados en una construcción, pero no más que aquellos que consideran que esta expresión significa solamente el rechazo de la monarquía hereditaria. 


\subsection{Los tipos de indeterminación}

RG define el escepticismo ante las reglas como la idea, fundada sobre la indeterminación del derecho, según la cual es necesariamente falsa toda teoría que afirme que no existe más que una, y sólo una, interpretación correcta de un texto.

La idea de que la interpretación está dirigida a reducir la indeterminación, es decir, a reducir las dudas acerca del significado de los textos es, a decir verdad, bastante extraña. Tomada literalmente, equivaldría a la afirmación "in claris cessat interpretatio", mientras que bien sabemos que, para poder afirmar que un texto es claro, es necesario haberlo interpretado. En realidad, esta idea parece incluso contradecir la definición misma de interpretación como "atribución de significado". Quien interpreta no afirma en todos los casos que lo hace porque tiene una duda. Por el contrario, generalmente, sobre todo cuando la interpretación no es acompañada por una justificación, el intérprete afirma que el texto es perfectamente claro y que posee el significado en cuestión.

Claramente se podría sostener que no se trata más que de un ejercicio retórico destinado a disimular la ausencia de argumentos o el carácter arbitrario de la decisión. Pero, ¿sobre qué base podemos afirmar que el texto interpretado es en realidad indeterminado, a pesar de la afirmación del intérprete de que el texto es claro? Ello no puede hacerse de otra forma más que invocando propiedades generales de los lenguajes naturales.

En realidad, el escepticismo ante las reglas bien puede perfectamente abandonar esta idea de la indeterminación de los textos. Es cierto que la teoría escéptica consiste, tal como lo dice RG, en la idea de que la one right answer thesis es falsa. Pero si esa tesis es falsa, no es porque los textos poseen numerosos significados. Es falsa simplemente porque el juez posee el poder de atribuir al texto un significado cualquiera y porque esa atribución no es jurídicamente cuestionable, lo que hace de ella una interpretación válida. Poco importa entonces que los textos aparezcan como indeterminados o unívocos antes de la interpretación. 


\section{Metateoría}

Bajo el título "metateoría" RG examina tres teorías de la interpretación jurídica, que reconstruye no como teorías psicológicas (dirigidas a determinar si la interpretación es un acto de conocimiento o de voluntad), sino como teorías semánticas, es decir, teorías acerca del valor de verdad de los enunciados interpretativos.

2.1. A decir verdad, la oposición psicológica-semántica no parece aquí de gran utilidad. Las teorías que presentan la interpretación jurídica como un acto de conocimiento o de voluntad no son efectivamente teorías psicológicas, en el sentido de que pretendan describir procesos psicológicos reales. Cuando se sostiene que la interpretación es un acto de voluntad, no se pretende afirmar que el sujeto que interpreta es conciente de ejercitar su voluntad. No habría ningún inconveniente en admitir que, desde un punto de vista psicológico, un juez aplica real y sinceramente ciertos métodos para atribuir a un texto un cierto significado, que el cree así "describir", y sostener, a la vez, que la interpretación es un acto de voluntad, puesto que el resultado al cual el juez llega, la atribución de un significado, no posee valor de verdad. De todos modos, la teoría de la interpretación como acto de voluntad es únicamente una teoría semántica en el sentido de RG.

Pero eso no es lo esencial. Lo esencial reside en la crítica que, desde un punto de vista metateórico, RG dirige a las tres teorías señaladas: el noble sueño, la pesadilla, y una teoría intermedia, la vigilia. Su crítica está principalmente dirigida contra la teoría del significado que cada una de ellas presupone.

Resumamos: las dos teorías del noble sueño y de la pesadilla admiten ambas, afirma RG, que la interpretación está dirigida al conocimiento del significado. Es por ello que ellas presuponen un significado susceptible de conocimiento. Pero este significado no puede ser ni el significado literal, ni la intención del legislador, ya que no es posible determinar ni el uno ni la otra de otro modo más que describiendo las prácticas interpretativas de una comunidad de 
intérpretes. Si ello es así, entonces la descripción de un significado no es otra cosa más que la reconstrucción de estas prácticas y la teoría del significado no se distingue en nada de una teoría de la interpretación.

No podemos más que compartir estas críticas. Pero uno se espera que RG se sume a la tercera teoría, la teoría de la pesadilla, es decir, la teoría escéptica en su forma radical, según la cual los intérpretes auténticos pueden atribuir a los textos no importa qué significado que de todos modos producirá efectos jurídicos.

Ahora bien, si bien concede que esta teoría es verdadera en tanto que descripción del modo de funcionar de (la mayoría de) los sistemas jurídicos actuales $^{3}$, RG sostiene que ella no posee ninguna consecuencia en cuanto se refiere a los textos jurídicos, puesto que esa teoría se limita a afirmar que los intérpretes poseen el poder de facto de apartarse de los significados existentes. La teoría escéptica radical sostendría o presupondría -RG parece dudar acerca de este punto- que no existe ningún significado antes de la interpretación.

Una tesis de este tipo, dice RG, es insostenible:

- en efecto, si ella fuera verdadera, no sería posible distinguir entre las frases que tienen significado y las frases que no lo tienen,

- tampoco sería posible distinguir entre una verdadera interpretación y una creación; y una distinción de este tipo sería esencial.

En consecuencia, el escepticismo según RG puede resumirse en las tesis siguientes:

- siempre es posible interpretar un mismo texto de múltiples maneras diferentes,

- no existe ningún criterio de verdad para los enunciados interpretativos.

2.2. Me parece, sin embargo, que estas conclusiones son débiles.

2.2.1. El enfoque metodológico adoptado aquí por RG, consistente en el análisis de los presupuestos de cada una de estas teorías de la

${ }^{3}$ Ref. a texto de Guastini. 
interpretación jurídica y en la búsqueda de esos presupuestos en una concepción del significado, es perfectamente legítimo. No obstante,

a) las tres teorías no poseen la misma relación con la teoría del significado. Si bien es cierto que algunas teorías de la interpretación son cognoscitivas en el sentido de que presuponen, o bien que los enunciados poseen un único significado (como en el caso del noble sueño), o bien, según la teoría de la vigilia, que al menos en ciertos casos poseen un significado objetivo de modo tal que los enunciados interpretativos pueden tener valor de verdad. Por el contrario, no es cierto que la teoría escéptica radical, la pesadilla, presuponga, como RG afirma al menos en dos ocasiones, que los textos no poseen ningún significado antes de ser interpretados.

Esta teoría no es para nada la aplicación al derecho de una teoría general de la interpretación, sino una teoría autónoma. Ella se limita a constatar, observando el funcionamiento del derecho positivo, que desde un punto de vista jurídico, los enunciados poseen el significado que les es atribuido a través del proceso de interpretación. La afirmación de que los textos no poseen un significado jurídico antes de ser interpretados constituye, no el presupuesto de la teoría escéptica radical, sino una simple consecuencia de ella.

Además, esta teoría no afirma que los textos serían simplemente incomprensibles antes de una interpretación oficial, lo que sería evidentemente absurdo puesto que somos capaces de comprender los más variados enunciados y de atribuirles uno o más significados, sino simplemente que el significado que le atribuimos no es una norma.

b) RG se aparta del punto de vista metateórico cuando le reprocha a la teoría radical su incapacidad para distinguir entre interpretación y creación. Este punto no constituye ya un presupuesto o un fundamento epistemológico de la teoría radical, sino simplemente una de sus tesis. Esta tesis puede ser verdadera o falsa, pero su valor de verdad no está atado a una teoría del significado.

c) Puede resultar sorprendente ver a RG rechazar una tesis que él mismo considera correcta en tanto que descripción del modo de funcionar de (la mayoría de) los sistemas jurídicos actuales, puesto que la principal -y quizás la única- cualidad que puede exigirse a una teoría de la 
interpretación jurídica no es que diga algo sobre la esencia de la interpretación, sino que permita describir correctamente el derecho positivo.

2.2.2. Con relación al fondo de la crítica dirigida contra la teoría radical, $R G$ concede que los jueces -y, debería agregarse, todos aquellos que, sin ser jueces, poseen el poder de dar una interpretación jurídicamente indiscutible- pueden dar a los textos el significado que deseen, pero ello significa simplemente, según RG, que los jueces "pueden de facto ignorar el significado pre-existente de los textos normativos".

Esta afirmación es doblemente extraña. Por un lado, si el intérprete, por ejemplo una corte suprema, puede dar a un enunciado el significado que quiera, no es algo que suceda de facto, sino que tiene lugar porque el intérprete ha recibido la competencia y porque el orden jurídico hace que sus decisiones posean efectos jurídicos. No se trata de una tesis sociológica sobre las prácticas de las cortes supremas. La teoría escéptica no afirma que de jure estas cortes deben elegir entre numerosos significados posibles, sino que, de facto, ellas pueden descartarlos y dar al texto cualquier significado. La teoría constata que el orden jurídico, al otorgar a un intérprete la última palabra, le otorga también el poder de dar a los textos el significado que quiera.

Es posible que, en la mayor parte de los casos, los jueces se ajusten a las prácticas interpretativas habituales de la comunidad de juristas a la que pertenecen, pero esta constatación no pertenece a la teoría del derecho si no a la sociología. La teoría del derecho no busca saber qué es lo que hacen los hombres, si no aquello que según el derecho deben hacer o aquello que poseen el poder de hacer, aun si usan ese poder raramente.

Por otro lado, es el propio RG quien se muestra cognitivista en este punto al afirmar que las cortes supremas pueden descartar un significado pre-existente. Ello lo compromete con aceptar que existe un significado de este tipo y que es cognoscible. Sin dudas, RG tiene en mente el significado que correspondería a las prácticas interpretativas de una comunidad de juristas, pero este significado no es el significado que el 
derecho positivo confiere a los textos, no es el significado con el que debemos tomar los textos.

¿RG es en definitiva un cognitivista? RG se preocupa por señalar que reconocer que existe un significado pre-existente es distinto de afirmar que existe un único significado, pero no precisa en qué consiste esa diferencia. Su posición parece similar a la de Kelsen (a quien se refiere oportunamente en el párrafo 1.1.), en cuanto este último distingue entre la interpretación científica y la interpretación auténtica y dice que la primera es una actividad de conocimiento. Podemos suponer que RG rechazaría admitir que es un cognitivista, no porque él llame cognitivista a quien piensa que existe un único significado pre-existente y no cognitivista a quien piensa que existen numerosos, sino porque dentro de su esquema los múltiples significados pre-existententes no son inherentes al texto, tal como lo quisiera la teoría del significado único, son solo significados posibles, correspondientes a las diferentes prácticas interpretativas usadas por una comunidad de juristas.

Esta defensa es perfectamente aceptable, pero me parece que entonces la teoría que nos es presentada no es una teoría de la interpretación jurídica, si por teoría de la interpretación jurídica entendemos una teoría que describe correctamente la naturaleza o los efectos de la interpretación en el derecho. En efecto, por mucho que sepamos que para cada texto existe una pluralidad de significados posibles, lo esencial es que el único significado, es decir la única norma válida es aquella que resulta de la decisión, cualquiera sea ésta, de una autoridad competente para interpretar.

RG dirá todavía que el conocimiento de los significados posibles es necesario para distinguir entre la genuina interpretación (la elección entre los significados posibles que se encuentran dentro del marco interpretativo) y la creación del derecho por el juez. Contra ello podemos alzar las siguientes objeciones:

- no existe ningún modo para redactar con certeza la lista de todos los significados posibles de un texto, de modo tal que sea posible afirmar que el significado elegido por el intérprete se ubica dentro o fuera del marco y que, en consecuencia, el intérprete ha llevado a 
cabo una genuina interpretación o, por el contrario, ha creado una nueva norma.

- Y aun suponiendo que ello fuera posible, no tendría ninguna consecuencia sobre la validez de la operación del intérprete, como tampoco existiría, desde un punto de vista jurídico, ninguna diferencia entre una norma resultado de una genuina interpretación y una norma resultado de un acto de creación.

La historia constitucional francesa ofrece un buen ejemplo. En 1962 el Presidente de la República, el general De Gaulle, consideró necesario llevar a cabo una reforma de la Constitución para que a partir de ese momento el Presidente de la República fuese elegido por el sufragio universal. Sin embargo, el artículo 89 de la Constitución establecía un proceso de reforma en dos etapas. El proyecto de reforma debía, en primer lugar, ser adoptado por las dos asambleas en términos idénticos y luego sometido o bien a la aprobación del Parlamento, o bien a referéndum. El senado disponía así de un poder de veto y, teniendo en cuenta su composición, era bastante probable que en 1962 lo habría ejercido. Siguiendo este procedimiento la revisión habría sido imposible. Pero la Constitución incluía también el artículo 11 que permitía al Presidente de la República someter directamente a referéndum un proyecto de ley "relativo a la organización de los poderes públicos". La casi totalidad de los juristas de la época consideraban que el artículo 11 no podía servir para reformar la Constitución. Sin embargo, esta fue la vía que De Gaulle utilizó, interpretando la expresión "organización de los poderes públicos" como sinónimo de "constitución". Si por "significados posibles" se entiende aquellos que se corresponden con las prácticas interpretativas de la comunidad de juristas, De Gaulle evidentemente se salía del marco, pero él fue el intérprete auténtico en la medida en que su decisión no podía ser jurídicamente discutida. El referéndum tuvo lugar y el resultado fue positivo, y todos los presidentes de la República han sido posteriormente elegidos por sufragio universal.

Tenemos entonces que elegir entre las siguientes opciones:

- Afirmar que el recurso al referéndum era inconstitucional y que, en consecuencia, la ley constitucional de 1962 no es válida y que ninguna elección llevada a cabo sobre la base de esa ley 
constitucional es válida, como tampoco es válido ninguno de los actos cumplidos por los presidentes que han sido elegidos a partir de ella. Todo lo cual sería claramente absurdo.

- Afirmar que, si bien no es válida, la reforma fue una revolución, un hecho o, en otros términos, que la ley constitucional del 62 entró en vigor sin ser válida. Sin embargo, más allá del hecho que la distinción entre vigor y validez se vería desprovista de toda consecuencia jurídica en cuanto las normas inválidas pero en vigor se aplicarían del mismo modo que las normas válidas, sería muy difícil, sino imposible, identificar aquellas normas. Además, respecto de cada una de esas normas sería posible encontrar teóricos que sostengan que se trata de normas inválidas puesto que el intérprete se ha salido del marco, y otros teóricos que sostengan que, por el contrario, se trata de normas perfectamente válidas porque el significado elegido era uno de los significados posibles. Es de temer que estas discrepancias de apreciación no serían más que el reflejo de las preferencias de cada uno de los teóricos, quienes se esforzarían por hacerlas prevalecer sobre las preferencias de los intérpretes auténticos. Esta actitud no es para nada diferente de aquella que consiste en llevar a cabo una evaluación moral sobre el derecho en vigor.

- Admitir simplemente que la interpretación válida es aquella que resulta de la decisión de un intérprete auténtico, cualquiera sea su contenido. Esto es precisamente a lo que puede resumirse, en mi opinión, la teoría escéptica. Es a partir de ello que se vuelve legítimo, desde un punto de vista positivista, describir y explicar las elecciones de los intérpretes sobre la base de sus preferencias, de los vínculos argumentativos que pesan sobre ellos y de las situaciones estratégicas en las que se encuentran.

\section{Conclusión}

Tal como bien lo dice RG, una teoría descriptiva de la interpretación jurídica no tiene ninguna necesidad de una teoría del significado, puesto 
que ella misma es una teoría del significado en el contexto jurídico (2.4.). Por lo tanto es necesario considerar las teorías, no desde un punto de vista metateórico, si por ello entendemos la búsqueda de sus fundamentos y de sus presupuestos, sino apreciarlas simplemente según su relación con la realidad de la que buscan dar cuenta. Se trata aquí de saber cuál es la teoría que da cuenta de mejor manera de la interpretación jurídica.

Ahora bien, me parece bien que, tal como es formulada por RG, la teoría escéptica no cumple satisfactoriamente con esta función. En efecto, no basta con afirmar que existe una pluralidad de interpretaciones posibles y que es el intérprete quien elige entre ellas. Tampoco alcanza con agregar que esto es lo que por lo general hacen los intérpretes. Es necesario precisar además qué es lo que dispone el derecho para los casos en los que el intérprete elige un significado que no se encuentra en la lista de significados posibles. La respuesta, cuando se trata de un intérprete de última instancia, es que el derecho dispone que ese significado, cualquiera sea, debe ser considerado como el único significado del texto. La teoría escéptica replanteada es, o bien una teoría normativa (ella indica el modo en el que debería comportarse el intérprete), o bien una teoría descriptiva, pero descriptiva de las prácticas más extendidas, es decir, una teoría sociológica o, incluso, si afirma que la interpretación fuera del marco de los significados posibles no es válida, una teoría descriptiva del sistema jurídico, pero una teoría errónea. 